\title{
BMJ Open Rehabilitation via HOMe Based gaming exercise for the Upper-limb post Stroke (RHOMBUS): protocol of an intervention feasibility trial
}

\author{
Cherry Kilbride, ${ }^{1}$ Daniel J M Scott, ${ }^{1}$ Tom Butcher, ${ }^{1}$ Meriel Norris, ${ }^{1}$ \\ Jennifer M Ryan, ${ }^{1,2}$ Nana Anokye, ${ }^{1}$ Alyson Warland, ${ }^{1}$ Karen Baker, ${ }^{1}$ \\ Dimitrios A Athanasiou, ${ }^{3}$ Guillem Singla-Buxarrais, ${ }^{3}$ Alexander Nowicky ${ }^{4}$
}

To cite: Kilbride C, Scott DJM, Butcher T, et al. Rehabilitation via HOMe Based gaming exercise for the Upper-limb post Stroke (RHOMBUS): protocol of an intervention feasibility trial. BMJ Open 2018;8:e026620. doi:10.1136/ bmjopen-2018-026620

- Prepublication history and additional material for this paper are available online. To view please visit the journal (http:// dx.doi.org/10.1136/bmjopen2018-026620).

Received 11 September 2018 Revised 1 October 2018 Accepted 3 October 2018

Check for updates

C Author(s) (or their employer(s)) 2018. Re-use permitted under CC BY-NC. No commercial re-use. See rights and permissions. Published by BMJ.

${ }^{1}$ Ageing Studies Theme, Institute of Environment, Health and Societies, Brunel University, London, UK

${ }^{2}$ Department of Epidemiology and Public Health Medicine, Royal College of Surgeons in Ireland, Dublin, Ireland ${ }^{3}$ Neurofenix, London, UK ${ }^{4}$ Centre for Cognitive Neuroscience, College of Health and Life Sciences, Brunel University, London, UK

Correspondence to Dr Cherry Kilbride; Cherry.Kilbride@brunel.ac.uk

\section{ABSTRACT}

Introduction Effective interventions to promote upperlimb recovery poststroke are characterised by intensive and repetitive movements. However, the repetitive nature of practice may adversely impact on adherence. Therefore, the development of rehabilitation devices that can be used safely and easily at home, and are motivating, enjoyable and affordable is essential to the health and well-being of stroke survivors. The Neurofenix platform is a nonimmersive virtual reality device for poststroke upper-limb rehabilitation. The platform uses a hand controller (a NeuroBall) or arm bands (NeuroBands) that facilitate upper-limb exercise via games displayed on a tablet. The Rehabilitation via HOMe Based gaming exercise for the Upper-limb post Stroke trial aims to determine the safety, feasibility and acceptability of the Neurofenix platform for home-based rehabilitation of the upper-limb poststroke. Methods and analysis Thirty people poststroke will be provided with a Neurofenix platform, consisting of a NeuroBall or NeuroBands (dependent on impairment level), seven specially designed games, a tablet and handbook to independently exercise their upper limb for 7 weeks. Training commences with a home visit from a research therapist to teach the participant how to safely use the device. Outcomes assessed at baseline and 8 weeks and 12 weeks are gross level of disability, pain, objectively measured arm function and impairment, self-reported arm function, passive range of movement, spasticity, fatigue, participation, quality of life (QOL) and health service use. A parallel process evaluation will assess feasibility, acceptability and safety of the intervention through assessment of fidelity to the intervention measured objectively through the Neurofenix platform, a postintervention questionnaire and semistructured interviews exploring participants' experiences of the intervention. The feasibility of conducting an economic evaluation will be determined by collecting data on $\mathrm{QOL}$ and resource use.

Ethics and dissemination Ethics approval granted from Brunel University London (10249-MHR-

Mar/2018-12322-2). Trial results will be submitted for publication in journals, presented at national and international conferences and distributed to people with stroke.

Trial registration number ISRCTN60291412; Pre-results.
Strengths and limitations of this study

- The Rehabilitation via HOMe Based gaming exercise for the Upper-limb post Stroke trial will investigate the feasibility, acceptability and safety of a novel gaming platform (the Neurofenix platform) at home for upper-limb exercise after stroke.

- Upper-limb activity data will be objectively measured by the device. Assessment outcome measures include objective (assessed blind to timepoint) and self-reported measures.

- To be maximally inclusive, stroke survivors with moderate to severe arm impairment will be included in the study.

- The feasibility of conducting an economic evaluation will be determined by collected data on quality of life and resource use.

- This is a home-based intervention study; thus, participants and researchers collecting the data will not be blinded.

\section{INTRODUCTION}

Stroke is the leading cause of severe disability worldwide with approximately 17 million new strokes each year. ${ }^{2}$ The UK has 1.2 million stroke survivors with 110000 first-time strokes occurring each year resulting in an estimated societal cost of $£ 26$ billion per year. $^{12}$ Following stroke, $85 \%$ of people initially experience upper-limb weakness, and of those with minimal movement on hospital admission, only $11 \%-14 \%$ regain full function of their arm. ${ }^{2-4}$ This loss in upper-limb function results in increased dependence and decreased quality of life (QOL). ${ }^{5}$ Reduced upper-limb function has been identified as a strong predictor of lowered psychological well-being poststroke. ${ }^{56}$ Innovation and investigation of effective treatments for arm recovery has been identified as a priority for stroke research. ${ }^{7}$ 
Evidence indicates the most effective interventions to improve upper-limb function are characterised by high intensity and repetitive practice. ${ }^{8}$ A higher intensity and frequency of upper-limb stroke rehabilitation is associated with improved QOL, ${ }^{9}$ motor function and ability to perform activities of daily life ${ }^{10}$ and is cost-effective. ${ }^{11}$ The UK quality standard for stroke advises $45 \mathrm{~min}$ of each relevant therapy for a minimum of 5 days a week. ${ }^{11}$ However, a 2015 UK national stroke audit showed on average most hospitals are unable to meet this quality standard. ${ }^{12}$ Specifically, time spent retraining the upper limb is very low, with an average of 32 repetitions per rehabilitation session. ${ }^{13}{ }^{14}$ As such, there is a growing emphasis on the stroke survivor exercising independently without the presence of a therapist. However, adherence to home exercise is known to be poor. ${ }^{1516}$ A perceived lack of support and feedback along with boredom with exercises are the most frequently cited factors associated with poor compliance. ${ }^{1718}$

Virtual reality (VR)-based activities have been suggested as an intervention to improve upper-limb recovery by providing motivating environments or gameplay to facilitate rehabilitation. ${ }^{19}$ This digital health solution helps address boredom and compliance problems, can facilitate increased time in therapy and may not be reliant on therapist contact time. ${ }^{19}{ }^{20}$ In addition, the ability of VR activities to provide feedback may enhance motor learning. ${ }^{2122}$ Visual feedback via an on-screen character (avatar) can activate mirror neurones, which may aid recovery from stroke. $^{2324}$

VR can be considered in terms of the level of immersion provided, that is, the degree the user feels present in the virtual world due to the technical aspects of the VR environment. Immersive systems can generate life-scaled, three-dimensional images, with surround sound auditory and sensory feedback such as vibration, and pressure, ${ }^{25}$ whereas non-immersive systems involve two-dimensional images typically viewed on a screen with interaction being via controller-based systems (such as computer keyboards, joysticks, balance boards and handheld devices) or via camera-based tracking systems. ${ }^{26}$ Non-immersive systems are more commonly used for rehabilitation as they have smaller space requirements, cost less and have fewer side effects (eg, motion sickness). ${ }^{27}$

The Neurofenix platform is a non-immersive device designed to enable and encourage stroke survivors to independently exercise their upper limb with minimal therapist input. The platform was developed by Neurofenix, a bioengineering enterprise (www.neurofenix. com), along with stroke survivors and neurological physiotherapists. The platform consists of a hand controller or armbands, seven specially designed games, a tablet and an instruction handbook.

\section{Study aims and objectives}

This study aims to determine the safety, feasibility and acceptability of the Neurofenix platform for home-based rehabilitation of the upper-limb poststroke. A secondary aim is to test procedures to inform a definitive randomised controlled trial to assess the clinical and cost-effectiveness of the Neurofenix platform with stroke survivors. The study objectives are:

1. To assess the safety, feasibility and acceptability of using the Neurofenix platform intervention at home for the rehabilitation of the upper limb after stroke.

2. To assess the feasibility of conducting a definitive trial of the clinical and cost-effectiveness of the Neurofenix platform intervention.

3. To understand the factors relating to people with stroke and the intervention that may impact on fidelity to the intervention.

\section{METHODS AND ANALYSIS \\ Trial design}

The Rehabilitation via HOMe Based gaming exercise for the Upper-limb post Stroke study is a non-randomised intervention trial (figure 1). A total of 30 participants will be recruited to use the Neurofenix platform at home for 7 weeks ( 1 week training, 6 weeks exercise). Assessments will be performed at baseline and 8 and 12 weeks. A parallel process evaluation will assess the safety, feasibility and acceptability of the intervention and the feasibility of conducting a definitive trial. Semistructured interviews will be used to explore the perspectives of participants receiving this complex intervention.

\section{Study setting}

The Department of Clinical Sciences, Brunel University London will coordinate the study. Assessments will be conducted, and the intervention delivered in participants' homes.

\section{Trial status}

At the time of submission of this study protocol, data collection is ongoing.

\section{Participants}

Inclusion criteria

- Aged 18 or over

- Capacity to consent

- Self-reported diagnosis of stroke (unilateral haemorrhagic or ischaemic)

- 12 weeks minimum poststroke and finished formal rehabilitation for their arm, that is, National Health Service (NHS) or private provider

- Mild to severe reduction in arm function poststroke, estimated by a Motricity Index ${ }^{28}$ score between 9 and 25 for elbow and shoulder movement

- Able to sit or stand independently (using an aid if necessary) for a minimum of $5 \mathrm{~min}$

- Can communicate in English, that is, sufficient for completion of trial intervention and assessment

Exclusion criteria

- Unstable medical conditions

- Uncontrolled photosensitive epilepsy 


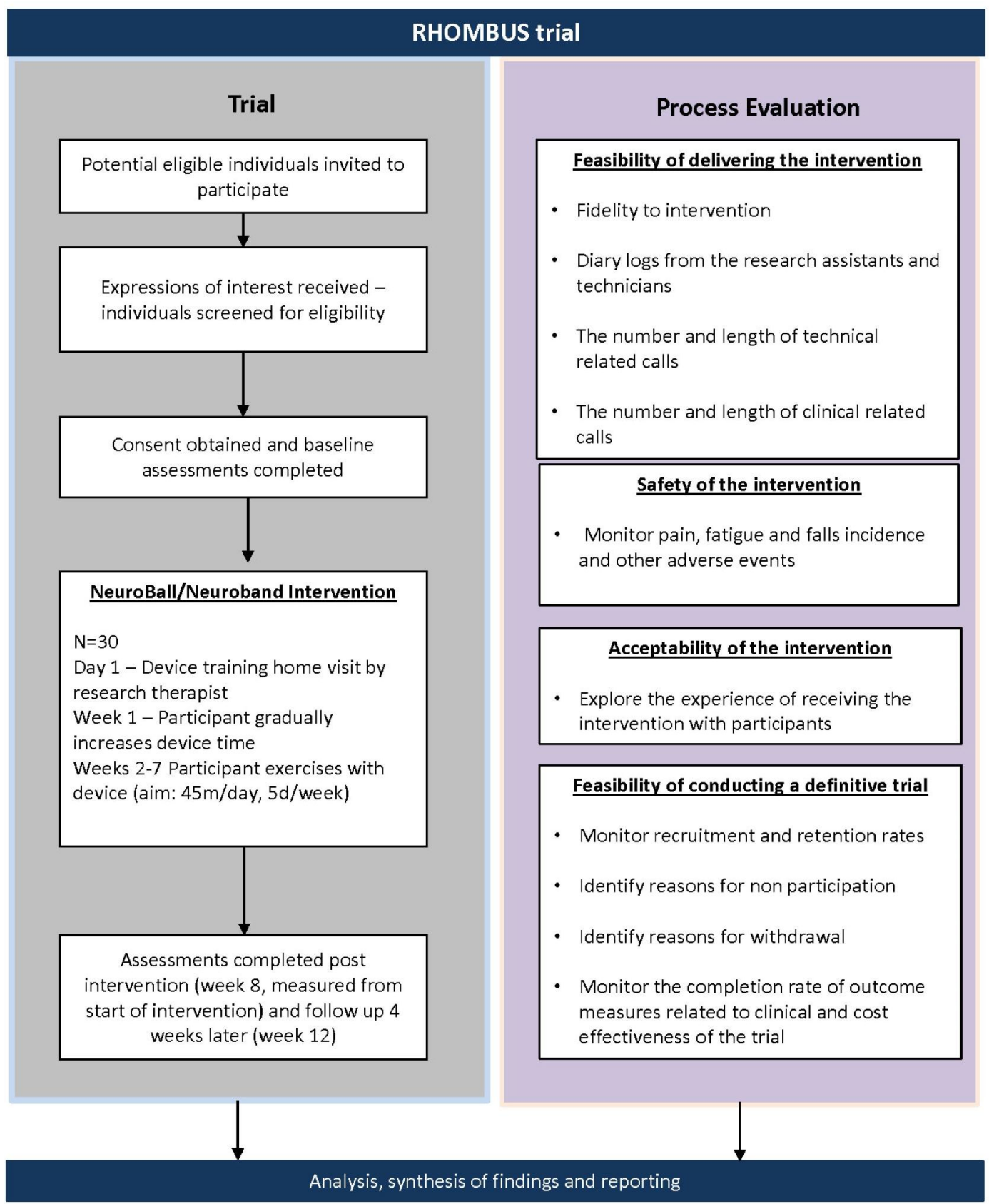

Figure 1 Rehabilitation via HOMe Based gaming exercise for the Upper-limb post Stroke (RHOMBUS) trial design.

- Acquired brain injury from other causes, bilateral or cerebellar lesions

- Uncompensated visual neglect, hemianopia or uncorrected visual field deficits (assessed by the National Institutes of Health Stroke Scale) ${ }^{29}$

- Pre-existing, unremitting arm pain at rest

\section{Sample size}

This is a feasibility study and no power calculation for the primary outcome(s) is required. The primary analytic aim is to evaluate the safety, feasibility and acceptability via the process evaluation data.

\section{Recruitment}

Participants will be recruited from Brunel's established database of people with stroke who have consented to contact about future stroke research studies and the ISRCTN Registry website. Participants will also be recruited through Different Strokes and the Action for Rehabilitation in Neurological Injury Institute. These participants will be accessed via gatekeepers and informed written consent will be taken by research therapists (see online supplementary appendices A, B and C for examples of the informed consent materials used). 
Recruitment will run from April 2018 to August 2018. Thirty participants will be recruited over 5 months, equating to approximately 1.5 participants per week.

\section{Reasons for non-participation}

A non-participation questionnaire will be distributed to those who do not wish to participate in the study. This will help identify reasons for refusal and any differences in baseline characteristics between participants and non-participants.

\section{Intervention}

The Neurofenix platform is a portable non-immersive VR device for gamification of upper-limb stroke rehabilitation. The platform uses either a hand controller, the NeuroBall or armbands, NeuroBands, to promote specific practice of movements in the shoulder, elbow, wrist and/ or hand through uniquely designed games displayed on a tablet. To ensure adequate reporting of the intervention a Template for Intervention Description and Replication checklist was completed (table 1).

This study will examine the Neurofenix platform intervention over a 7-week period, commencing with a home visit from a research therapist. The participant will be given the Neurofenix platform (see table 1 for content details) and trained how to use their device independently or with the help of a carer (if requested). The participant will then be advised to use the platform with an aim of $45 \mathrm{~min}$ a day, 5 days a week or more if they are able, self-limiting use based on fatigue and pain, and slowly increasing their use of the device over the first week. Participants will be advised to contact the research therapists as necessary for clinical and technical support throughout the intervention. A more detailed description of the intervention process can be found in table 1 .

\section{Assessments}

Assessments will be completed in the participant's home at baseline and 8 and 12 weeks postintervention commencement. All participants will be requested to complete follow-up assessments, including those who withdraw or were withdrawn from the intervention. Those participants will also be asked to complete a questionnaire to identify reasons for withdrawal. The assessment lasts approximately 2 hours and regular breaks will be given to mitigate fatigue and burden. In addition to the stated outcomes, further information will be collected on at baseline on socioeconomic status, stroke and relevant medical history and lifestyle factors.

\section{Outcomes}

All outcome measures follow a standardised operating procedure.

\section{Objectively measured arm function}

Arm function will be objectively assessed using the Action Research Arm Test (ARAT). The ARAT assesses upper-limb function using observational methods and is divided into four subtests of grasp, grip, pinch and gross arm movement. Performance on each item is rated on a four-point ordinal scale from 0 to 3 with a maximum score of 57, a higher score indicating a better level of function. The ARAT has excellent inter-rater reliability in chronic stroke populations ${ }^{30-32}$ and excellent test-retest reliability, moderate construct validity and responsiveness. ${ }^{30}$ The ARAT has a minimally clinically important difference (MCID) in people with chronic stroke of 5.7 points, equivalent to roughly $10 \%$ of the measure's total range. ${ }^{31}$ The ARAT has been shown to have floor and ceiling effects and a moderate burden. ${ }^{30}$ Completion of the ARAT at each timepoint will be videoed for blinded assessor scoring at a later date.

\section{Objectively measured arm impairment}

The Fugl-Meyer Assessment-upper limb (FMA-UL) will assess arm impairment. Performance is rated on a threepoint ordinal scale from 0 to 2 , with a maximum score of 66 , a higher score indicates minimal or no impairment. The FMA-UL has excellent inter-rater reliability when used in the chronic stroke population. ${ }^{33}$ MCID ranges from 4.25 to 7.25 depending on different facets of upperlimb movement. ${ }^{34}$

\section{Passive range of movement}

Passive range of movement of the upper limb will be assessed for the shoulder, elbow, wrist, thumb and index finger using goniometry to increase the inter-rater reliability of these measurements. ${ }^{35}$

\section{Spasticity}

The Modified Modified Ashworth Scale (MMAS) will assess for spasticity. The MMAS tests resistance to passive movement of a joint with varying degrees of velocity. Performance is rated on a six-point ordinal scale from 0 to 5 with a higher score indicating higher spasticity. ${ }^{36}$ The MMAS has good to very good intra-rater and inter-rater reliability for the elbow and wrist flexors. ${ }^{37} 38$

\section{Self-reported arm function}

Self-reported arm use will be assessed using the 28-item Motor Activity Log (MAL). The MAL is a semistructured interview where individuals are asked to rate the amount of movement during 28 daily functional tasks. The MAL has excellent test-retest reliability in chronic stroke patients. ${ }^{39}$ The MCID is $1.0-1.1{ }^{40} \mathrm{~A}$ higher score on the amount of use scale indicates the respondent's ability to use the stroke-affected arm is closer to their prestroke ability.

\section{Fatigue}

Fatigue will be assessed using the seven-item Fatigue Severity Scale (FSS-7). This questionnaire explores how fatigue interferes with stated activities, the participant rates the severity on a seven-point Likert scale for each item. The FSS-7 is recommended as it has better validity and reliability and is likely more sensitive for measuring change in fatigue in people with stroke. ${ }^{41}$ Minimum score 


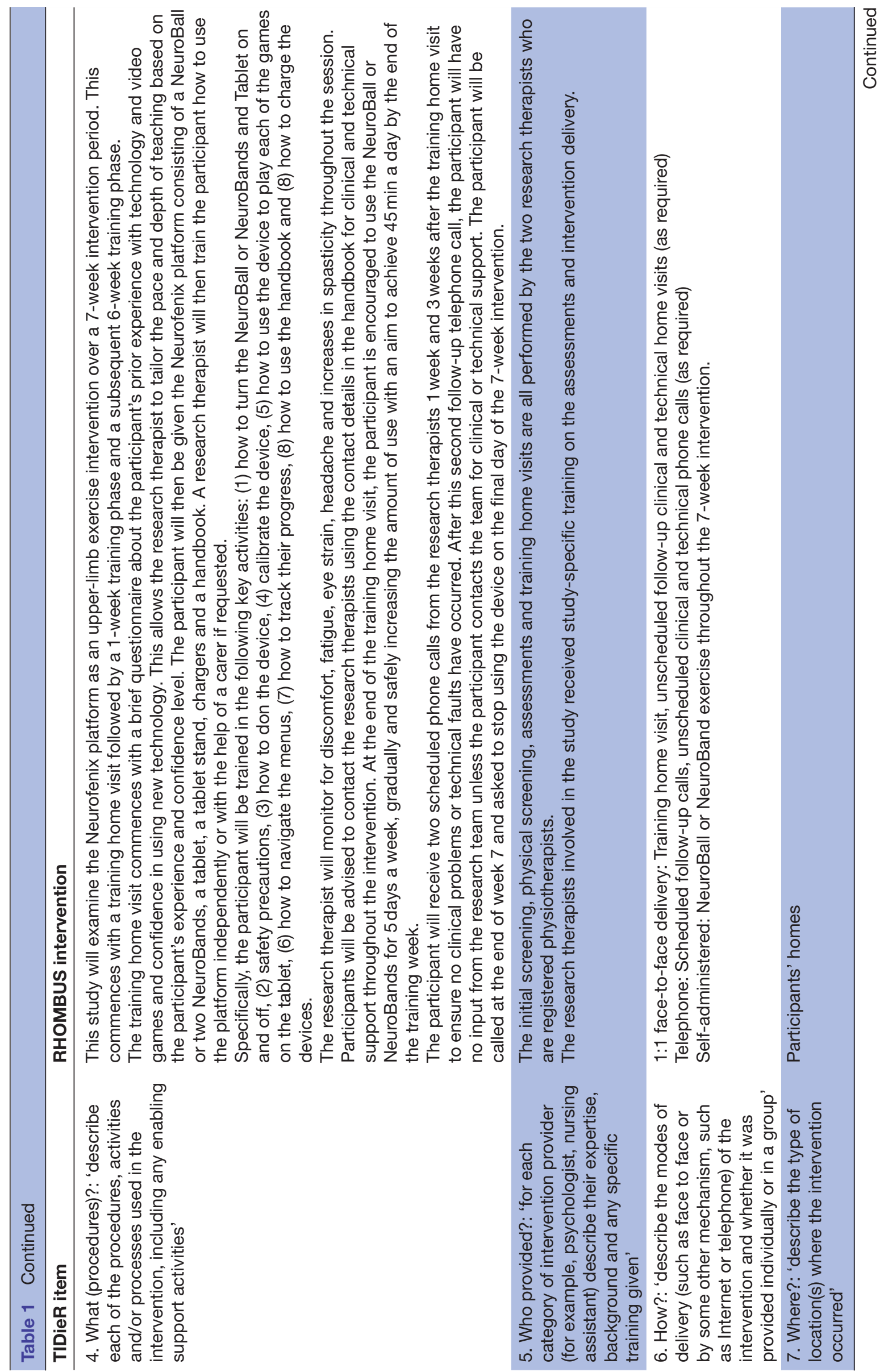




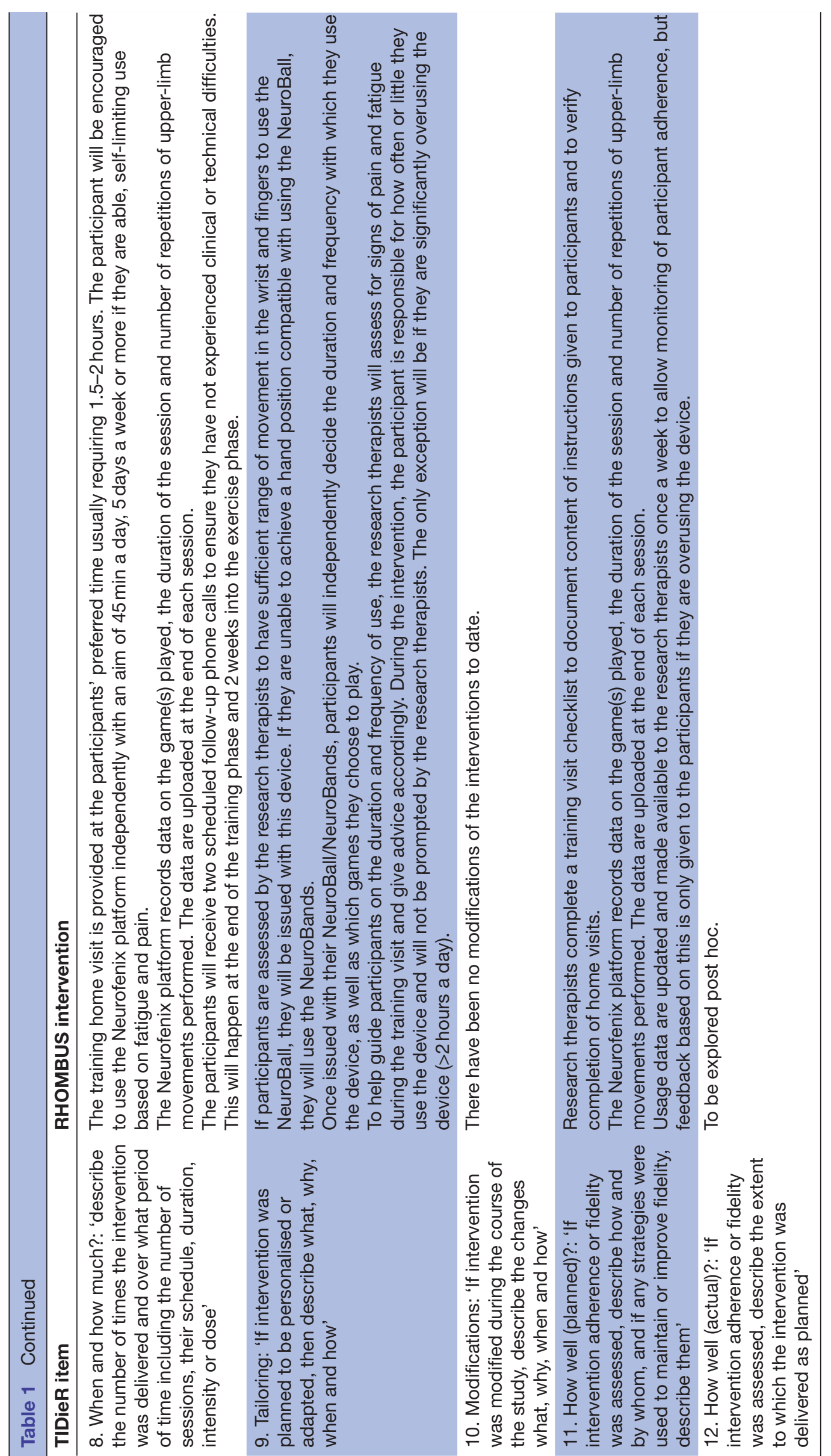


is 7 and maximum 49, a higher score indicates a greater impact of fatigue on a person's activities.

\section{Quality of life}

QOL will be assessed using the EuroQol 5 Dimensions 5 Levels (EQ-5D-5L). The EQ-5D-5L describes and values health in five dimensions: mobility, self-care, usual activities, pain/discomfort and anxiety/depression. ${ }^{42}$ Each dimension has five response categories ranging from no problems to extreme problems. Participants also rate their overall health on the day of the interview on a visual analogue scale from 0 to 100 (EuroQol-Visual Analogue Scale) ${ }^{42}$ In people with stroke, the EQ-5D-5L has been shown to have reasonable concurrent validity $(\rho=0.255-$ $0.703, \mathrm{p}<0.05$ ), acceptable responsiveness and a MCID of $0.10 .^{43}$

\section{Participation}

Participation will be assessed using the 10-item Subjective Index of Physical and Social Outcome (SIPSO). ${ }^{44}$ The SIPSO measures factors relating to physical functioning/ mobility and social/emotional functioning. The SIPSO is a valid and reliable measure of social and physical integration in people with stroke, ${ }^{44}$ with a higher internal construct validity when the subscales are used, instead of the total scale. ${ }^{45}$ Each question is scored from 0 to 4 ; minimum score of 0 and maximum of 40 , a higher score indicates an increased ability to reintegrate to a 'normal' lifestyle.

\section{Pain}

Pain will be assessed using a visual analogue scale (VAS) from 0 , no pain, to 10 , excruciating pain, over the last 7 days. A VAS is a valid measure of pain intensity and is responsive to change. ${ }^{4647}$ To help those with language or mild cognitive problems, the scale is illustrated with emotive faces.

\section{Gross level of disability}

The simplified modified Rankin Scale questionnaire (smRSq) will be used to measure the participant's level of disability. The smRSq requires yes or no answers from a patient or caregiver. The smRSq has excellent reliability by telephone $(\kappa=0.76(0.63$ to 0.90$))$ and in person $(\kappa=0.71(95 \%$ CI 0.57 to 0.86$)$ ) and correlates with QOL and initial stroke severity. ${ }^{48} 49$

\section{Health service use}

A modified version of the Client Service Receipt Inventory (CSRI) will assess health service use. The CSRI collects retrospective information on service utilisation, service-related issues and income. ${ }^{50}$ The CSRI has been successfully used to estimate service use among adults with stroke. ${ }^{51}$

\section{Fidelity to use of the Neurofenix platform}

Fidelity to use of the Neurofenix platform will be assessed objectively using data collected via the sensors in the NeuroBall and NeuroBands and the software on the tablet. These automatically collect data on the time spent actively exercising in each game and the number of movement repetitions.

\section{Clinical and technical support provided}

The amount of clinical and technical support will be recorded by the research therapists: the number, length and content of all calls and visits with participants.

\section{Economic evaluation}

This study will assess the feasibility of conducting an economic evaluation alongside a definitive clinical effectiveness of the Neurofenix platform. The aim of this feasibility study is to examine the practicality of collecting resource use and QOL data, the quality of the data and the amount of missing data observed. Resource use will include the following: therapist training; training home visits; clinical and technical support; participants' out-ofpocket expenses related to any additional exercise being undertaken; and health and personal social service use. These will be collected through diaries, management records, questionnaires and interviews.

In the definitive trial, the economic evaluation will take NHS, personal social services and participants' perspective. ${ }^{52}$ The main outcome of the economic analysis will be an incremental cost per quality-adjusted life-year (QALY), based on EQ-5D-5L. Unit costs will be taken from the NHS reference costs (eg, DH 2016), standard unit costs (eg, PSSRU 2015) and published literature.

\section{Process evaluation}

A parallel process evaluation will be conducted alongside the trial to determine the feasibility, acceptability and safety of the intervention. The feasibility of delivering the intervention will be assessed through multiple mechanisms following the guidelines for fidelity in complex rehabilitation interventions. ${ }^{53}$ Specifically, feasibility and perceived adequacy of training the research therapists will be assessed by analysis of their field notes. Feasibility of delivering the training session, clinical or technical calls and visits, and carrying out assessments will be determined by number, length and content of training sessions, the clinical and technical call and visit logs and therapists' field notes on assessment and intervention participant burden.

The feasibility and acceptability of the intervention for people with stroke will be assessed by evaluating the distribution of fidelity to the intervention, as measured by the Neurofenix platform, in terms of duration of active game play. Associations between participant-related factors, such as level of impairment, and fidelity will be explored. In addition, participant-reported experience will be investigated using the pretraining and post-training questionnaires, postintervention questionnaire, and by conducting semistructured interviews, as described next.

\section{Pretraining and post-training questionnaire}

Each participant will complete a pretraining questionnaire prior to the training session to explore previous 
experience with technology and gaming and to measure confidence levels with new technology. A post-training questionnaire will be completed after the training session to assess their confidence level with using the Neurofenix platform and to explore how the participant might use the device in the following weeks.

\section{Postintervention questionnaire}

A postintervention questionnaire will be conducted after completion of the intervention period to assess the participant's perception of the Neurofenix platform and its components.

\section{Interviews}

Semistructured interviews will be conducted with a purposive sample of 18 participants to explore the perspectives of those receiving the intervention. Sampling will involve key criteria such as gender, age, device used (NeuroBall or the NeuroBands), amount of use (use of the device 4 or more days a week pragmatically classified as high use, 3 or less as low use), level of upper-limb impairment and function (ARAT scores $0-9$ as severe, $10-21$ as moderately severe, $22-43$ as moderate, $43-53$ as mild and $55-57$ as full).$^{54}$

Interviews will be conducted at the participant's house by a researcher trained in qualitative research methods. Topic guides developed from relevant literature and the specific aims of the process evaluation will be used. Where possible, the participant will be interviewed by a research therapist not involved in the intervention training to reduce the risk of socially desirable responses.

\section{Safety}

Pain and fatigue will be assessed at baseline, 8 weeks and 12 weeks. Self-reported pain will be assessed using a pain VAS and asking the average pain over the last week, the section relating to pain in the EQ-5D-5L. Fatigue will be assessed using the FSS-7. The number of episodes of pain, falls, fatigue, eye strain and other reported adverse events will be collated to assess the safety of the intervention. Research therapists will proactively enquire about changes in the participant's health or any compromises of safety since the last contact. A record of the incidence of adverse events from baseline measures until the end of the trial for each participant will be maintained. Falls incidence will be determined by asking participants at each contact point if they have fallen or tripped since the last contact. Although this method of assessing falls relies on the recall ability of the participant, it accurately detects injurious falls in community-dwelling older adults. ${ }^{55} \mathrm{An}$ adverse event is considered serious if it results in death, is life-threatening, requires hospitalisation or prolongation of existing hospitalisation and results in persistent or significant disability or incapacity. Participants who experience a serious adverse event will be withdrawn from the study.

A summary of all data collected and when these are collected is provided in table 2.

\section{Data management}

Personal data collected during the trial will be handled and stored in accordance with the 1998 Data Protection Act and the General Data Protection Regulation 2018. Consent forms will be kept separate from other data in site trial master files at Brunel University London in a locked, secure environment. To preserve participant anonymity, only their allocated trial number and initials will be recorded on trial documentation (except the consent form). Confidentiality of all participant data will be maintained and information by which a participant could be identified will not be disclosed to a third party. Only non-identifiable clinical data will be shared with Neurofenix. Neurofenix will automatically collect data from the Neurofenix platform including user name, active game play and number of movement repetitions. Data generated by the Neurofenix platform will adhere to a Data Privacy Protocol, informed by Information Commissioner's Office guidelines and is compliant with the General Data Protection Regulation.

Use of study data will be controlled by the principal investigator. All data and documentation related to the trial will be stored in accordance with applicable regulatory requirements and access to data will be restricted to authorised trial personnel.

Qualitative interviews will be audio recorded and will be stored electronically and identified by trial number only. Transcripts will be anonymised or assigned a pseudonym; files will be stored using password-protected files. The ARAT video recordings will be stored electronically on encrypted and password-protected devices and identified by trial number only. Pseudonymised quantitative data will be made available in a public repository following publication of findings.

\section{Patient and public involvement}

People with stroke have been involved in the ongoing development of the intervention, including 18 stroke survivors who participated in an earlier study examining the usability of the Neurofenix platform. Two additional stroke survivors have provided input to the protocol, reviewed trial documentation, including participant information sheets, participant invite letters and questionnaires. The stroke survivors will continue to advise the research team throughout the trial, including dissemination of the results.

\section{Data analysis}

\section{Qualitative data}

To determine the feasibility, acceptability and safety of the intervention with people poststroke, interviews will be analysed using framework analysis. ${ }^{56}$ This method provides a strong audit trail of the analytical process, which enhances transparency. ${ }^{57}$ The technique involves five iterative stages: familiarisation, identifying thematic framework, labelling, charting and mapping and interpretation, following which significant themes can be presented. ${ }^{57}$ As a further step to enhance rigour in this 
Table 2 Schedule of assessments and outcome measures

\begin{tabular}{|c|c|c|c|c|c|}
\hline Clinical assessments & Preintervention & Intervention & Eight weeks & Postintervention & Twelve weeks \\
\hline Informed consent & $x$ & & & & \\
\hline Sociodemographic measurement & $\mathrm{x}$ & & & & \\
\hline ARAT & $x$ & & $x$ & & $x$ \\
\hline FMA-UL & $x$ & & $x$ & & $\mathrm{x}$ \\
\hline PROM-UL & $x$ & & $x$ & & $x$ \\
\hline MAL & $\mathrm{x}$ & & $x$ & & $\mathrm{x}$ \\
\hline FSS-7 & $x$ & & $x$ & & $x$ \\
\hline SIPSO & $x$ & & $x$ & & $x$ \\
\hline smRSq & $x$ & & $x$ & & $\mathrm{x}$ \\
\hline Pain VAS & $x$ & & $x$ & & $\mathrm{x}$ \\
\hline EQ-5D-5L & $x$ & & $x$ & & $x$ \\
\hline CSRI & $\mathrm{x}$ & & $x$ & & $\mathrm{x}$ \\
\hline Training and training questionnaire & & $x$ & & & \\
\hline Neurofenix platform use & & $x$ & & & \\
\hline Clinical and technical support & & $x$ & & & \\
\hline Postintervention questionnaire & & $x$ & & $\mathrm{x}$ & \\
\hline Semistructured interview & & $x$ & & $x$ & \\
\hline Falls & & $x$ & $x$ & $\mathrm{x}$ & $\mathrm{x}$ \\
\hline Upper-limb pain & & $x$ & $x$ & $x$ & $x$ \\
\hline $\mathrm{AE}$ and $\mathrm{SAE}$ & & $\mathrm{x}$ & $\mathrm{X}$ & $\mathrm{X}$ & $\mathrm{x}$ \\
\hline
\end{tabular}

AE, adverse event; ARAT, Action Research Arm Test; CSRI, Client Service Receipt Inventory; FMA-UL, Fugl-Meyer Assessment-upper limb; FSS-7, seven-item Fatigue Severity Scale; MAL, Motor Activity Log; PROM-UL, passive range of movement-upper limb; SAE, serious adverse event; SIPSO, Subjective Index of Physical and Social Outcome; smRSq, simplified modified Rankin Scale questionnaire; VAS, visual analogue scale; EQ-5D-5L, EuroQol 5 Dimensions 5 Levels.

process, three researchers will independently code the same three transcripts and then meet to discuss and agree on codes assigned to each passage and their definitions.

\section{Quantitative data}

Distribution of the data will be examined using histograms, Q-Q plots and cross-tabulations. Descriptive statistics will be used to report pain and fatigue at baseline and follow-up, the number of participants experiencing adverse events during follow-up and the number of adverse events per participant. Generalised estimating equations will be used to examine change in pain and fatigue across timepoints.

Descriptive statistics will be used to report all data relating to fidelity, feasibility and acceptability of the intervention including the number and duration of clinical and technical calls, average session length and time spent in each game, enjoyment of games and number of episodes of pain experienced during intervention. Recruitment, retention and outcome measure completion will be described using frequencies and percentages.

Descriptive statistics will be used to report key participant characteristics across levels of fidelity to the intervention in terms of frequency and duration of active game play. Participant characteristics will include level of disability, pretraining confidence in using new technology, post-training confidence in using the NeuroBall/ Bands, level of support provided to use the NeuroBall/ Bands and age.

The practicality, quality of data, quantity of missing data and reasons for missing data associated with the data collection tools will be recorded as part of determining the feasibility of conducting a phase three trial.

\section{Timeline}

The trial is funded for a period of 14 months and commenced in January 2018. Recruitment commenced in April 2018 and will be completed in August 2018. The final follow-up assessment is projected to be completed in October 2018 with data analysis and report writing being conducted from October onwards.

\section{Ethics}

The study is sponsored by Brunel University London and will be conducted in accordance with the approved protocol. Any protocol modifications will be notified to the Brunel University London Ethics Committee and consent will be reobtained from participants if required. The principles of the Declaration of Helsinki and Good Clinical Practice guidelines will be adhered to, along with 
UK legislation and Brunel University Research integrity guidance.

\section{Monitoring}

As the intervention is low risk and the potential harm is not anticipated, there will be no Data Monitoring Committee, interim analyses or stopping rules.

\section{Administrative structures}

The trial will be run by the principal investigator, coinvestigators and two research therapists. The Chair of the College Research Ethics Committee will provide additional trial oversight, along with quarterly monitoring meetings with the funder and Neurofenix. Financial accounts will be externally audited.

\section{Dissemination}

The dissemination plan will be developed in the early phases of the trial and will involve social media, broadcast media, the internet and electronic mail as well as more traditional routes, that is, peer-reviewed journal and national and international conferences. Publications will follow the Enhancing the Quality and Transparency of Health Research guidelines for reporting non-randomised studies. Authorship will follow international guidelines (International Committee of Medical Journal Editors criteria). The results will be disseminated to all participants and to those who wanted to participate but did not meet the inclusion criteria and who agreed to be contacted for research purposes.

Acknowledgements The authors would like to thank the two stroke survivors who assisted the development of the intervention and study materials. Further thanks to the group facilitators of Different Strokes and the Action for Rehabilitation from Neurological Injury. Thanks to Professor Christina Victor for her continuing support.

Contributors All authors listed meet the International Committee of Medical Journal Editors criteria for authorship. CK and GSB conceived the study, DJMS, TB, AN, JMR, NA, AW and MN designed the study. GSB, DAA, DJMS, TB and CK designed the intervention. CK will lead the running of the trial. DJMS and TB will lead the collection, management and analysis of the data. KB will lead blinded ARAT assessment. MN will lead the process evaluation. JMR will lead the statistical analysis. NA will lead the evaluation of the feasibility of the economic evaluation. All authors have read and approved the final manuscript.

Funding This work was supported by Innovate UK grant number 104188[3463]. The study sponsor is Brunel University London. Contact: Professor Peter Hobson, University Research Ethics Committee Chair (peter.hobson@brunel.ac.uk), Brunel University London, London.

Disclaimer Neurofenix has no influence on the design of the study, data collection, analysis and interpretation of the data and in writing the manuscript.

Competing interests GSB and DAA are employed by Neurofenix (UK), a company that manufactures and markets home-based training solutions. Neurofenix will provide the Neurofenix platforms and technical support to the research therapists.

\section{Patient consent Obtained.}

Ethics approval Ethics approval Ethical approval has been granted by the College of Health and Life Sciences Research Ethics Committee (REC) in Brunel University London (10249-MHR-Mar/2018-12322-2).

Provenance and peer review Not commissioned; peer reviewed for ethical and funding approval prior to submission.

Data sharing statement Pseudonymised data will be made available in a public repository once the data have obtained validation through publication.

Open access This is an open access article distributed in accordance with the Creative Commons Attribution Non Commercial (CC BY-NC 4.0) license, which permits others to distribute, remix, adapt, build upon this work non-commercially, and license their derivative works on different terms, provided the original work is properly cited, appropriate credit is given, any changes made indicated, and the use is non-commercial. See: http://creativecommons.org/licenses/by-nc/4.0/.

\section{REFERENCES}

1. Ward NS. Restoring brain function after stroke - bridging the gap between animals and humans. Nat Rev Neurol 2017;13:244-55.

2. Stroke Association. State of the nation stroke statistics. 2018 https://www.stroke.org.uk/resources/state-nation-stroke-statistics

3. Kwakkel G, Kollen BJ. Predicting activities after stroke: what is clinically relevant? Int J Stroke 2013;8:25-32.

4. Nakayama $\mathrm{H}$, Jørgensen HS, Raaschou HO, et al. Recovery of upper extremity function in stroke patients: the Copenhagen Stroke Study. Arch Phys Med Rehabil 1994;75:394-8.

5. Nichols-Larsen DS, Clark PC, Zeringue A, et al. Factors influencing stroke survivors' quality of life during subacute recovery. Stroke 2005;36:1480-4.

6. Wyller TB, Sødring KM, Sveen U, et al. Are there gender differences in functional outcome after stroke? Clin Rehabil 1997;11:171-9.

7. Pollock A, St George B, Fenton M, et al. Top 10 research priorities relating to life after stroke-consensus from stroke survivors, caregivers, and health professionals. Int J Stroke 2014;9:313-20.

8. Langhorne P, Coupar F, Pollock A. Motor recovery after stroke: a systematic review. Lancet Neurol 2009;8:741-54.

9. Veerbeek JM, van Wegen E, van Peppen R, et al. What is the evidence for physical therapy poststroke? A systematic review and meta-analysis. PLoS One 2014;9:e87987.

10. Sehatzadeh S. Effect of increased intensity of physiotherapy on patient outcomes after stroke: an evidence-based analysis. Ont Health Technol Assess Ser 2015;15:1.

11. Dworzynski K, Ritchie G, Fenu E, et al. Rehabilitation after stroke: summary of NICE guidance. BMJ 2013;346:f3615.

12. Royal College of Physicians. Clinical effectiveness and evaluation unit on behalf of the intercollegiate stroke working party. Sentinel Stroke National Audit Programme (SSNAP) Clinical audit August-November 2016 public report, 2016.

13. Lang CE, MacDonald JR, Gnip C. Counting repetitions: an observational study of outpatient therapy for people with hemiparesis post-stroke. J Neurol Phys Ther 2007;31:3-10.

14. Hayward KS, Brauer SG. Dose of arm activity training during acute and subacute rehabilitation post stroke: a systematic review of the literature. Clin Rehabil 2015;29:1234-43.

15. Donoso Brown EV, Dudgeon BJ, Gutman K, et al. Understanding upper extremity home programs and the use of gaming technology for persons after stroke. Disabil Health J 2015;8:507-13.

16. Jurkiewicz MT, Marzolini S, Oh P. Adherence to a home-based exercise program for individuals after stroke. Top Stroke Rehabil 2011;18:277-84.

17. Hendrie W. The dog ate my trainers: Synapse, 2011.

18. Tijou I, Yardley L, Sedikides C, et al. Understanding adherence to physiotherapy: findings from an experimental simulation and an observational clinical study. Psychol Health 2010;25:231-47.

19. Laver KE, Lange B, George S, et al. Virtual reality for stroke rehabilitation. Cochrane Database Syst Rev 2017;31.

20. Turolla A, Dam M, Ventura L, et al. Virtual reality for the rehabilitation of the upper limb motor function after stroke: a prospective controlled trial. J Neuroeng Rehabil 2013;10:85.

21. Subramanian SK, Lourenço CB, Chilingaryan G, et al. Arm motor recovery using a virtual reality intervention in chronic stroke: randomized control trial. Neurorehabil Neural Repair 2013;27:13-23.

22. Kiper P, Piron L, Turolla A, et al. The effectiveness of reinforced feedback in virtual environment in the first 12 months after stroke. Neurol Neurochir Pol 2011;45:436-44.

23. Celnik P, Stefan K, Hummel F, et al. Encoding a motor memory in the older adult by action observation. Neuroimage 2006;29:677-84

24. Franceschini M, Ceravolo MG, Agosti M, et al. Clinical relevance of action observation in upper-limb stroke rehabilitation: a possible role in recovery of functional dexterity. A randomized clinical trial. Neurorehabil Neural Repair 2012;26:456-62.

25. Man D. Common issues of virtual reality in neuro-rehabilitation. Virtual reality: InTech, 2010.

26. Anderson F, Annett M, Bischof WF. Lean on Wii: physical rehabilitation with virtual reality Wii peripherals. Stud Health Technol Inform 2010;154:229-34.

27. Prashun P, Hadley G, Gatzidis C, et al. Investigating the trend of virtual reality-based stroke rehabilitation systems. Information Visualisation (IV), 2010 14th International Conference; IEEE 2010. 
28. W Bohannon R. Motricity index scores are valid indicators of paretic upper extremity strength following stroke. Journal of Physical Therapy Science 1999;11:59-61.

29. Goldstein LB, Samsa GP. Reliability of the National Institutes of Health Stroke Scale: extension to non-neurologists in the context of a clinical trial. Stroke 1997;28:307-10.

30. Sivan M, O'Connor RJ, Makower S, et al. Systematic review of outcome measures used in the evaluation of robot-assisted upper limb exercise in stroke. J Rehabil Med 2011;43:181-9.

31. Van der Lee JH, De Groot V, Beckerman H, et al. The intra- and interrater reliability of the action research arm test: a practical test of upper extremity function in patients with stroke. Arch Phys Med Rehabil 2001;82:14-19.

32. Yozbatiran N, Der-Yeghiaian L, Cramer SC. A standardized approach to performing the action research arm test. Neurorehabil Neural Repair 2008;22:78-90.

33. Duncan PW, Propst M, Nelson SG. Reliability of the Fugl-Meyer assessment of sensorimotor recovery following cerebrovascular accident. Phys Ther 1983;63:1606-10.

34. Page SJ, Fulk GD, Boyne P. Clinically important differences for the upper-extremity Fugl-Meyer Scale in people with minimal to moderate impairment due to chronic stroke. Phys Ther 2012;92:791-8.

35. van de Pol RJ, van Trijffel E, Lucas C. Inter-rater reliability for measurement of passive physiological range of motion of upper extremity joints is better if instruments are used: a systematic review. $J$ Physiother 2010;56:7-17.

36. Bohannon RW, Smith MB. Interrater reliability of a modified Ashworth scale of muscle spasticity. Phys Ther 1987;67:206-7.

37. Gregson JM, Leathley MJ, Moore AP, et al. Reliability of measurements of muscle tone and muscle power in stroke patients. Age Ageing 2000;29:223-8.

38. Gregson JM, Leathley M, Moore AP, et al. Reliability of the Tone Assessment Scale and the modified Ashworth scale as clinical tools for assessing poststroke spasticity. Arch Phys Med Rehabil 1999;80:1013-6.

39. van der Lee JH, Beckerman $\mathrm{H}$, Knol DL, et al. Clinimetric properties of the motor activity log for the assessment of arm use in hemiparetic patients. Stroke 2004;35:1410-4.

40. Simpson LA, Eng JJ. Functional recovery following stroke: capturing changes in upper-extremity function. Neurorehabil Neural Repair 2013;27:240-50.

41. Lerdal A, Kottorp A. Psychometric properties of the Fatigue Severity Scale-Rasch analyses of individual responses in a Norwegian stroke cohort. Int J Nurs Stud 2011;48:1258-65.

42. Herdman M, Gudex C, Lloyd A, et al. Development and preliminary testing of the new five-level version of EQ-5D (EQ-5D-5L). Qual Life Res 2011;20:1727-36.

43. Chen P, Lin KC, Liing RJ, et al. Validity, responsiveness, and minimal clinically important difference of EQ-5D-5L in stroke patients undergoing rehabilitation. Qual Life Res 2016;25:1585-96.
44. Trigg R, Wood VA. The Subjective Index of Physical and Social Outcome (SIPSO): a new measure for use with stroke patients. Clin Rehabil 2000;14:288-99.

45. Kersten P, Ashburn A, George S, et al. The subjective index for physical and social outcome (SIPSO) in stroke: investigation of its subscale structure. BMC Neurol 2010;10:26.

46. Jensen MP, Karoly P, Braver S. The measurement of clinical pain intensity: a comparison of six methods. Pain 1986;27:117-26.

47. Ferreira-Valente MA, Ribeiro JL, Jensen MP, et al. Coping with chronic musculoskeletal pain in Portugal and in the United States: a cross-cultural study. Pain Med 2011;12:1470-80.

48. Bruno A, Akinwuntan AE, Lin C, et al. Simplified modified rankin scale questionnaire: reproducibility over the telephone and validation with quality of life. Stroke 2011;42:2276-9.

49. Bruno A, Shah N, Akinwuntan AE, et al. Stroke size correlates with functional outcome on the simplified modified Rankin Scale questionnaire. J Stroke Cerebrovasc Dis 2013;22:781-3.

50. Beecham J, Knapp M. Costing psychiatric interventions. Measuring mental health needs 2001;2:200-24.

51. Rodgers H, Shaw L, Cant R, et al. Evaluating an extended rehabilitation service for stroke patients (EXTRAS): study protocol for a randomised controlled trial. Trials 2015;16:205

52. NICE. Developing NICE guidelines: the manual. 2014. https://www. nice.org.uk/process/pmg20/chapter/introduction-and-overview

53. Poltawski L, Norris M, Dean S. Intervention fidelity: developing an experience-based model for rehabilitation research. J Rehabil Med 2014;46:609-15

54. Hoonhorst $\mathrm{MH}$, Nijland $\mathrm{RH}$, van den Berg JS, et al. How do FuglMeyer arm motor scores relate to dexterity according to the action research arm test at 6 months poststroke? Arch Phys Med Rehabil 2015;96:1845-9.

55. Ganz DA, Higashi T, Rubenstein LZ. Monitoring falls in cohort studies of community-dwelling older people: effect of the recall interval. $J$ Am Geriatr Soc 2005;53:2190-4.

56. Ritchie J, Lewis J, Nicholls CM, et al. Qualitative research practice: a guide for social science students and researchers: Sage, 2013.

57. Holloway I, Galvin K. Qualitative research in nursing and healthcare: John Wiley \& Sons, 2016.

58. Kleim JA, Jones TA. Principles of experience-dependent neural plasticity: implications for rehabilitation after brain damage. J Speech Lang Hear Res 2008;51:S225.

59. Rand D, Givon N, Weingarden $\mathrm{H}$, et al. Eliciting upper extremity purposeful movements using video games: a comparison with traditional therapy for stroke rehabilitation. Neurorehabil Neural Repair 2014;28:733-9.

60. Brunner I, Skouen JS, Hofstad H, et al. Is upper limb virtual reality training more intensive than conventional training for patients in the subacute phase after stroke? An analysis of treatment intensity and content. BMC Neurol 2016;16:219. 